\title{
Thin-Layer Solutions of the Helmholtz Equation
}

\author{
J R Ockendon \\ Mathematical Institute, University of Oxford, \\ Oxford, OX2 6GG, UK \\ R H Tew \\ School of Mathematical Sciences, University of Nottingham, \\ University Park, Nottingham, NG7 2RD, UK
}

August 19, 2020

\begin{abstract}
This paper gives a brief overview of some configurations in which highfrequency wave propagation modelled by Helmholtz equation gives rise to solutions that vary rapidly across thin layers. The configurations are grouped according to their mathematical structure and tractability and one of them concerns a famous open problem of mathematical physics.
\end{abstract}

\section{Introduction}

High-frequency asymptotics in the frequency domain has been studied intensively in St Petersburg for many decades, and much of the early work is reviewed in [19]. Meanwhile, the idea of applying systematic boundary layer theory in this area was pioneered in [2]. This paper is a sequel to [1], upon which we will rely for much of the background and detailed arguments.

Boundary layer approximations to the two-dimensional Helmholtz equation

$$
\nabla^{2} \phi+k^{2} \phi=0
$$

arise when the complex potential $\phi$ represents modulated plane waves with large real wavenumber $k$. The simplest ansatz is to write $\phi \sim e^{i k x} A$, where the complex amplitude $A$ has an asymptotic expansion in inverse powers of $k$, with $x \sim k^{-\lambda_{1}}, y \sim k^{-\lambda_{2}}$ and $1+\lambda_{1}=2 \lambda_{2}$. To lowest order, this gives the so-called parabolic wave equation

$$
2 i \frac{\partial A}{\partial x}+\frac{\partial^{2} A}{\partial y^{2}}=0
$$

This is a complexification of the convection/conduction equation for heat flow in a thermal boundary layer. As we will see, (2) can be used to model a twodimensional beam of light in the same way that the real version of (2) can model 
a plume of heat. However, we caution that solutions of (2) will hardly ever be uniform approximations to solutions of (1), especially if $x$ and/or $y$ has infinite range. Moreover, there are many more intricate asymptotic representations for thin layers, such as the Friedlander-Keller expansion

$$
\phi \sim e^{i k x+i k^{1 / 3} u(x, y)} A(x, y ; k),
$$

where $A$ no longer satisfies (2) to lowest order (see [3]) and we will see how such expansions arise naturally in Section 3.

A simple fundamental solution of (2) takes the form

$$
A=e^{-2 i\left[\lambda\left(y-y_{0}\right)+\lambda^{2}\left(x-x_{0}\right)\right]},
$$

where $\lambda, x_{0}, y_{0}$ are constants with $\lambda$ being a Fourier transform variable, and we will see that superposition of such functions with weight $f(\lambda)$ lead to many useful exact solutions of (2). Another fundamental solution is the Green's function or point source solution

$$
A=\left(x-x_{0}\right)^{-1 / 2} e^{i\left(y-y_{0}\right)^{2} / 2\left(x-x_{0}\right)},
$$

which can be obtained by taking $f(\lambda)=1$ and integrating (4) along the real $\lambda$ axis with an appropriate definition of $\left(x-x_{0}\right)^{1 / 2}$. We note that when $x_{0}=y_{0}=$ 0 in (4) and $f(\lambda)$ is fairly general, the resulting superposition is closely related to weighting (5) with $g\left(y_{0}\right)$ and setting $x_{0}=0$; indeed, $f$ and $g$ are Fourier transforms of each other. Almost all the solutions we will list in Sections 2 to 4 will involve superpositions of (4) and (5).

We remark that since (2) is invariant under effectively the same one-parameter groups as the heat equation, we can refer to [4] to note, for example, that if $A(x, y)$ satisfies (2), then so does the function $e^{-\lambda y+i \lambda^{2} x / 2} A(x, y+i \lambda x)$.

We will begin in Section 2 by briefly reviewing some well-known solutions of (2) that are localised away from any boundaries in the $(x, y)$ plane. These have all been described in some detail in [1] and we will only emphasise aspects that are relevant to Sections 3 and 4. Then, in Section 3, we will consider thin layers that are adjacent to prescribed boundaries and hence can be part of "modal solutions" in closed domains. This Section will build up to the "Fock-Leontovich" solution for (2) that models irradiation of a parabola which is locally tangent to the $x$-axis by a plane wave propagating along this axis. This problem acts as a paradigm for the one to be discussed in Section 4, namely the long-standing question of diffraction of a whispering gallery wave at an inflection point of a boundary. This is the so-called "Popov" problem which has attracted the attention of many researchers since the pioneering paper [5]. We will also make some conjectures about the solution in the light of recent numerical evidence. 


\section{Thin Layers with no Boundaries}

\subsection{Gaussian Beams}

No superposition is needed to model a Gaussian beam. All we need to do is set $x_{0}=i X_{0}$ with $X_{0}$ real, large and positive in (5), so that $A$ is a Gaussian beam with amplitude proportional to $\exp \left(-\left(y-y_{0}\right)^{2} / 2 X_{0}\right)$, at least for $|x| \ll X_{0}$, $\left|y-y_{0}\right| \sim X_{0}^{1 / 2}$. However, any such representation fails to be a uniformly valid approximation to a solution of (1) when $x \rightarrow \infty$. As described in [1], such beams spread laterally and eventually match with a multiple of the outgoing wave solution of (1) given by $\frac{\partial}{\partial x} H_{0}^{(1)}\left(k \sqrt{x^{2}+y^{2}}\right)$, where $H_{0}^{(1)}$ is the Hankel function of the first kind, which decays like $\left(x^{2}+y^{2}\right)^{-1 / 4}$. Many of the thin layers to be described later suffer from such nonuniformity. ${ }^{1}$

\subsection{Shadow Boundaries}

We can either seek a similarity solution of (2) of the form $A=A(y / \sqrt{x})$ or weight (4) with a function proportional to $\lambda^{-1}$ and integrate with respect to $\lambda$ over a suitable contour in the complex $\lambda$-plane to deduce that

$$
\int_{-\infty}^{y / \sqrt{2 x}} e^{i t^{2}} d t=\sqrt{\pi} e^{i \pi / 4}\left(1-\frac{1}{\sqrt{\pi}} e^{-i \pi / 4} \operatorname{Fr}(y / \sqrt{2 x})\right),
$$

where $F r$ is the Fresnel integral, also satisfies (2); it tends to $\sqrt{\pi} e^{i \pi / 4}$ as $y \rightarrow$ $+\infty$ (the illuminated region) and to zero as $y \rightarrow-\infty$ (the shadow) and its $y$-derivative is proportional to a Gaussian beam for appropriate values of $x$. We will see in Section 3 that the birth of a shadow boundary can be a complicated matter when it is initiated by a grazing ray.

\subsection{Thin Layers in Regions Bounded by an Ellipse}

We now describe two solutions of (2) that decay as $|y| \rightarrow \infty$ as in a Gaussian beam but are both components of a high-frequency eigensolution of the Helmholtz equation in an ellipse. Hence they are called modes, even though they do not comply with a boundary condition at the ellipse. They arise from superimposing solutions of the form (5) rewritten as

$$
(x+2 i)^{-1 / 2} \exp \left(-2 i(\lambda+i y / 2)^{2} /(x+2 i)\right)
$$

with a weighting $\lambda^{m} e^{-\lambda^{2} / 2}$, which gives the solution

$$
A=\left(x^{2}+4\right)^{-1 / 4} e^{i\left(y^{2} x / 2\left(x^{2}+4\right)-i(m+1 / 2) \tan ^{-1}(x / 2)\right)} D_{m}\left(\frac{2 y}{\left(x^{2}+4\right)^{1 / 2}}\right),
$$

\footnotetext{
${ }^{1}$ We remark that Gaussian beams also describe the thin layers that are present near a flat plate lying along the positive $x$-axis when it is irradiated by a field $e^{i k x}$, assuming the boundary conditions are other than Neumann conditions. However they are not uniformly valid near the origin.
} 
where the parabolic cylinder function $D_{m}(z)=2^{-m / 2} e^{-y^{2} / 4} H_{m}(y / \sqrt{2})$ and $H_{m}$ is the Hermite polynomial of degree $m$. We note that as $|y| \rightarrow \infty$,

$$
|A| \sim\left(\frac{y}{\left(x^{2}+4\right)^{1 / 4}}\right)^{m} e^{-y^{2} /\left(x^{2}+4\right)} .
$$

As described in [1] this solution can be "modalised" by taking hints from the Mathieu functions that arise when Helmholtz' equation is solved by separating the variables in elliptic coordinates to give

$$
A=\frac{\sqrt{c}}{\left(c^{2}-x^{2}\right)^{1 / 4}}\left(\frac{c+x}{c-x}\right)^{\frac{m}{2}+\frac{1}{4}} \exp \left(-i y^{2} x / 2\left(c^{2}-x^{2}\right)\right) D_{m}\left(\frac{e^{i \pi / 4} \sqrt{2 c} y}{\left(c^{2}-x^{2}\right)^{1 / 2}}\right),
$$

where $c$ is real and positive. This "focussing mode" represents a thin layer solution of (2) that lies close to the major axis of an ellipse whose foci are at $x= \pm c$. This phenomenon of a "terminating beam" is associated with the focusing of rays emanating from the ends of the major axis of the ellipse and it shows that small perturbations of perfect foci need not necessarily lead to the caustics to be described shortly.

If we replace $c$ by $i c$ in (8) we retrieve the solution

$$
\begin{aligned}
A & =\frac{\sqrt{c}}{\left(c^{2}+x^{2}\right)^{1 / 4}} \exp \left(-i y^{2} x / 2\left(c^{2}+x^{2}\right)-i(m+1) \tan ^{-1}\left(\frac{x}{c}\right)\right) \\
& \times D_{m}\left(\frac{y \sqrt{2 c}}{\left(c^{2}+x^{2}\right)^{1 / 2}}\right) .
\end{aligned}
$$

This represents a thin layer that lies along and terminates at the ends of the minor axis and it results from the concentration of rays that bounce repeatedly at the boundary; hence (9) is called a "bouncing ball mode". In fact there exist many more thin layer solutions of (2) that arise at the boundaries of the regions within which the modes in an ellipse have real rays. These boundaries are not straight and this leads us to study thin layers with non-zero curvature.

\subsection{Caustics}

Caustics are familiar thin layers because of their observability in, say, coffee cups on a sunny day. They model the smooth transition that occurs at the envelopes of the rays that underpin geometrical optics and hence they are inevitably curved. Thus it is convenient to rewrite (1) in orthogonal curvilinear coordinates and, to do this, we assume for simplicity that the envelope $y=f(x)$ is smooth and has no vertical tangents. Then we let $s$ measure the distance from some fixed point on the envelope to the point $(X, f(X))$ where $X$ is such that $f^{\prime}(X)(y-f(X))+x-X=0$, and $n$ is a coordinate along the normal whose distance from the envelope is $\left((y-f(X))^{2}+(x-X)^{2}\right)^{\frac{1}{2}}$; the sign of the 
square root is such that $y$ increases when $n$ increases and vice versa. Elementary geometry gives that the metrics in $(x, y)$ and $(s, n)$ are related by

$$
d x^{2}+d y^{2}=d n^{2}+(1-\kappa n)^{2} d s^{2},
$$

where $\kappa(s)$ is the curvature of the envelope, which is positive when the normal $n>0$ passes through the centre of curvature; we also assume $n<\kappa^{-} 1$. In these coordinates, Helmholtz equation becomes

$$
\begin{gathered}
\frac{1}{(1-n \kappa)}\left(\frac{\partial}{\partial n}\left((1-n \kappa) \frac{\partial \phi}{\partial n}\right)+\frac{\partial}{\partial s}\left(\frac{1}{(1-n \kappa)} \frac{\partial \phi}{\partial s}\right)\right)+k^{2} \phi= \\
\left(\frac{1}{(1-n \kappa)^{2}} \frac{\partial^{2}}{\partial s^{2}}+\frac{\partial^{2}}{\partial n^{2}}+\frac{n \kappa^{\prime}}{(1-n \kappa)^{3}} \frac{\partial}{\partial s}-\frac{\kappa}{(1-n \kappa)} \frac{\partial}{\partial n}+k^{2}\right) \phi=0,
\end{gathered}
$$

in contrast to the notation in [1], [10]. If there are no rapid variations in the $s$-direction and $n$ is small, $\phi$ is proportional to $e^{ \pm i k s}$ to lowest order.

However, there are much more interesting solutions in which $\phi \sim e^{i k s} A(s, n)$ as $k \rightarrow \infty$. In this case, to lowest order

$$
\frac{\partial^{2} A}{\partial n^{2}}-2 k^{2} n \kappa A=0,
$$

so that, with $n=k^{-2 / 3} N$, the physically realistic solution for $A$ is a multiple, which may depend on $s$, of the Airy function

$$
\operatorname{Ai}\left((2 \kappa)^{1 / 3} N\right)=\frac{1}{2 \pi i} \int_{-\infty}^{\infty} \exp \left(i(2 \kappa)^{1 / 3} N \lambda+i \lambda^{3} / 3\right) d \lambda,
$$

the shadow region being $N \rightarrow-\infty$ when $\kappa>0$. This result was derived systematically in [24], [23]. Also it is shown in [1] that (12) can be written in local Cartesian coordinates $(X . Y)$ near $s=0$ as a superposition of plane waves given by $(2)$. The result is proportional to

$$
\int_{-\infty}^{\infty} \exp \left(-i \lambda Y-i \lambda^{2} X / 2-i \kappa(0) \lambda^{3} / 6\right) d \lambda
$$

where $Y-\frac{1}{2} \kappa(0) X^{2}=N=k^{\frac{2}{3}} n, X=S=k^{1 / 3} s$. However the change in metric introduces a pre-factor in the relation between $e^{i k s}$ and $e^{i k x}$.

We will now evaluate the multiple of the Airy function that determines the enhanced amplitude at a caustic. To do this, we must match (12) with the ray expansion corresponding to a prescribed remote source of illumination with $s, n$ of $O(1)$. The technical complications that arise have been described in detail in [2], where a different coordinate system from ours is employed, and in [19]; hence we will only summarise the basic ideas here. For definiteness we take $\kappa>0$ so that the real rays are in $n<0$. Near the caustic where $s^{*},|n| \sim \frac{1}{2} \kappa\left(s^{*}\right)\left(s^{*}-s\right)^{2}$, ray theory reveals that $\phi$ comprises fields of the form

$$
\frac{I_{ \pm}}{\left|s^{*}-s\right|^{\frac{1}{2}}} \exp \left(i k\left(s^{*} \pm \frac{2}{3}\left(2 \kappa\left(s^{*}\right)^{\frac{1}{2}}(-n)^{\frac{3}{2}}\right)\right)\right)
$$


where $I_{ \pm}$are the amplitudes of the incoming and outgoing fields respectively, the former being prescribed. In order for a superposition of these fields to yield a function proportional to (12) as $N=k^{-\frac{2}{3}} n \rightarrow \infty$, we recall that $A i(-z) \sim \pi^{-\frac{1}{2}}(-z)^{-\frac{1}{4}} \sin \left(\frac{2}{3}(-z)^{\frac{3}{2}}+\frac{\pi}{4}\right)$ as $z \rightarrow-\infty$. Hence we infer that

(i) $I_{+}=-e^{\frac{i \pi}{2}} I_{-}$, which reflects the fact that there is a phase change of $\frac{\pi}{2}$ as a ray changes from being incoming to outgoing and

(ii) the Airy function in (12) is multiplied by the factor

$$
\frac{1}{2} I_{+}\left(s^{*}\right) k^{\frac{1}{6}} \pi^{\frac{1}{2}}\left(2 \kappa\left(s^{*}\right)\right)^{\frac{1}{3}} e^{i k s^{*}-\frac{i \pi}{4}},
$$

which reveals that the caustic amplifies the field by $k^{\frac{1}{6}}$.

We emphasise that both these results are consequences of the fact that $\phi$ is exponentially small inside the caustic.

\subsection{Cusped Caustics}

The commonest singularity that can occur in a caustic is a cusp described locally by, say, $|y|=\left(\frac{2}{3} x\right)^{3 / 2}, x>0$. Indeed, this cusp can arise when we consider the envelope of Gaussian beams, as described in [1]. To analyse this situation and those in the following sections, we therefore consider solutions of (11) that are localised near a curve

$$
y+\frac{\gamma}{m} x^{m}=0
$$

in Cartesian coordinates, with $m$ greater than 1. Then, for small $x, y, s$ and $n$ we can write

$$
\begin{aligned}
s & \sim x+O\left(x^{m}\right), \\
n & \sim y+\frac{\gamma}{m} x^{m}+o\left(x^{m}\right), \\
\kappa & \sim-\gamma(m-1) x^{m-2}+o\left(x^{m-2}\right) .
\end{aligned}
$$

Hence, when we again write $\phi=e^{i k s} A(s, n)$, we find that the dominant terms in (11) for large $k$ are, after those of $O\left(k^{2}\right)$ have been removed, those arising from the first two terms.

If we assume $n \sim O\left(k^{\alpha}\right), s \sim O\left(k^{\beta}\right)$ where $\alpha, \beta$ are negative and analogous to the parameters $\lambda_{1}, \lambda_{2}$ introduced after (1), then $\kappa \sim k^{(m-2) \beta}$ and the relevant terms balance when

$$
k^{1-\beta} \sim k^{\alpha+\beta(m-2)+2} \sim k^{2 \alpha} .
$$

Thus $\alpha=m /(1-2 m), \beta=1 /(1-2 m)$ and, to lowest order, (11) reduces to

$$
\frac{\partial^{2} A}{\partial n^{2}}+2 i \frac{\partial A}{\partial s}+2(m-1) \gamma n s^{m-2} A=0,
$$


where, for the rest of this section, $n, s$ are scaled variables. For our cusped caustics, $m=3 / 2, \gamma=\left(\frac{2}{3}\right)^{1 / 2}$ and $n$ and $s$ scale with $k^{-3 / 4}$ and $k^{-1 / 2}$ respectively, as described in more detail in [1]. Since $A$ decays as $|n| \rightarrow \infty$, we can take the Fourier transform $\tilde{A}=\int_{-\infty}^{\infty} A e^{i \lambda n} d n$ to give

$$
-\lambda^{2} \tilde{A}+2 i \frac{\partial \tilde{A}}{\partial s}+i \gamma s^{-1 / 2} \frac{\partial \tilde{A}}{\partial \lambda}=0 .
$$

The characteristic equations are

$$
\frac{d s}{2}=\frac{d \lambda}{\gamma s^{-1 / 2}}=\frac{i}{\lambda^{2}} \frac{d \tilde{A}}{\tilde{A}},
$$

so that the general solution for $\tilde{A}$ is

$$
\tilde{A}=f\left(\lambda-\frac{\gamma}{4} s^{1 / 2}\right) \exp \left[\frac{i}{6 \gamma^{2}}\left(\lambda^{4}+\gamma s^{1 / 2} \lambda^{3}\right)\right],
$$

where the function $f$ is arbitrary. In our case it is determined by matching as $s \rightarrow \infty$ with the upper/lower branches of the cusped caustic, which is described by (12) (further details of this matching are given in [1]). This means that $f$ is a constant and $A$ is thus proportional to

$$
\int_{-\infty}^{\infty} \exp \left[\frac{i}{6 \gamma^{2}}\left(\lambda^{4}+\gamma s^{1 / 2} \lambda^{3}\right)-i n \lambda\right] d \lambda
$$

We note that when we add a constant to $\lambda$ and make use of (15), we retrieve the Pearcey integral (2.89) of [1]. In the light of Section 3.1, we expect an increase in amplitude as the cusp is approached and the analysis in [1] shows that this increase is of $O\left(k^{\frac{3}{4}}\right)$.

As observed in [1], there is no noticeable thin layer near the negative $x$-axis, but there are thin layers of exponentially small amplitude around the Stokes lines $y= \pm\left(-\frac{2}{3} x\right)^{3 / 2}$. This is not surprising in view of the decay of the incoming Airy function (12).

In the next section we will consider the dramatic effect that modality can have on these solutions.

\section{Thin Layers Adjacent to Boundaries}

\subsection{The Birth of Creeping Waves}

As explained in Section 2.4, the asymptotic behaviour of solution (12) as $\kappa N \rightarrow$ $-\infty$ comprises two waves that can be matched to incoming and outgoing wave fields described by geometrical optics. The latter can be used to model the 
birth of a creeping wave field at a convex boundary at which $A$ or $\frac{\partial A}{\partial n}$ vanishes, depending whether the boundary is hard or soft. However, this cannot happen unless (12) is modified and this can be done by seeking waves that are weakly modulated in the $s$-direction in the form

$$
\phi \sim e^{i\left(k s+k^{\frac{1}{3}} u(s)\right)} A(s)
$$

it is shown in [10] that the lowest order terms in the asymptotic expansion for large $k$ preclude the function $u$ from depending on $n$. Then the leading order terms in (11) must be such that a balance occurs in which

$$
\frac{\partial^{2} A}{\partial n^{2}}+2\left(-k^{4 / 3} \frac{d u}{d s}-k^{2} n \kappa\right) A=0 .
$$

Hence we can satisfy boundary conditions on $N=0$ if we set $\frac{d u}{d s}=2^{-\frac{1}{3}} \kappa^{\frac{2}{3}} \sigma$, where, again with $n=k^{-\frac{2}{3}} N$,

$$
\frac{\partial^{2} A}{\partial N^{2}}+2\left(-\kappa N-2^{-\frac{1}{3}} \kappa^{\frac{2}{3}} \sigma\right) A=0
$$

and $\sigma$ is a positive zero of $A$ or $\frac{\partial A}{\partial N}$.

As remarked earlier, (19) is an example of a Friedlander-Keller ansatz, which will often be used throughout the rest of the paper. We remark that the creeping ray field for plane wave irradiation of a circular cylinder can be, with some effort, extracted from the explicit exact solution for arbitrary $k$ given in [6]. The amplitude of the creeping field turns out to be proportional to the one sixth power of the inverse of the radius of the circle, in contrast to the one third power that arises for a caustic.

\subsection{Whispering Gallery Waves}

When $\kappa>0$, the preceding approach applies to whispering gallery waves, for which the wave field as $N \rightarrow+\infty$ is exponentially small, of $O\left(e^{-\frac{2}{3}(2 \kappa)^{1 / 2} N^{3 / 2}}\right)$. The implications of (20) for the high-frequency spectrum for the Helmholtz equation in a closed region are discussed in [1]. We remark that only a singlyinfinite number of modes emerge at this lowest order of approximation and the two-dimensionality of the spectrum needs to be revealed by tracking the field around the boundary, assumed smooth and convex, and applying periodicity. The only reason we mention these waves explicitly here is because of interest in the behaviour of such waves near an inflection point where the boundary changes from being concave to convex, and this will be addressed in the final section. A stepping stone in this direction is the problem of grazing diffraction. 


\subsection{Grazing Diffraction and Fock-Leontovich Theory}

The problem of solving (1) subject to zero Dirichlet or Neumann data on a convex scatterer under plane wave irradiation has been studied extensively (see for example [7],[6]). It is convenient to subtract out the incident field $e^{i k x}$ and write

$$
\phi=\phi_{s c}+e^{i k x}
$$

and we will, for simplicity, only consider the case when

$$
\phi_{s c}=-e^{i k x}
$$

at the scatterer, whose local shape at the grazing point is parabolic. Our strategy is now to solve (16), with $m=2$, subject to $e^{i k s} A=-e^{i k x}$ on $y+\frac{1}{2} \gamma x^{2}=0$, where $x$ and $y$ have been scaled with $k^{-1 / 3}$ and $k^{-2 / 3}$, respectively. We also require $A \rightarrow 0$ as $\left(y+\frac{1}{2} \gamma x^{2}\right) \rightarrow+\infty$ and, crucially, that $A$ matches with the incoming specularly reflected field generated at the boundary for $x<0$. Thus we require

$$
\begin{gathered}
e^{i k s} A=-e^{i\left(k s-\gamma^{2} s^{3} / 6\right)} \\
\text { i.e. } A=-e^{-i \gamma^{2} s^{3} / 6} \text { on } y+\frac{1}{2} \gamma x^{2}=0,
\end{gathered}
$$

and that $e^{i k s} A$ must match with

$$
-\left(\frac{-x+\sqrt{x^{2}+3 y / 2 \gamma}}{3\left(x^{2}+3 y / 2 \gamma\right)^{1 / 2}}\right)^{1 / 2} e^{\left(i k\left(x+\frac{4}{27}\left(-x^{3}-\frac{9 x y}{4 \gamma}+\left(x^{2}+\frac{3 y}{2 \gamma}\right)^{3 / 2}\right)\right)\right)}
$$

as $x \rightarrow-\infty$ with $y+\frac{1}{2} \gamma x^{2}=O(1)$; this formula comes from a ray theory calculation as described in [1]. It shows that (22) applies as $s \rightarrow-\infty$ for $y+\frac{1}{2} \gamma x^{2}$ of $O(1)$.

The way is now open to again convert to inner curvilinear coordinates $S=$ $k^{\frac{1}{3}} s, N=k^{\frac{2}{3}} n$ and solve (16) by taking a Fourier transform in $s$ to give that

$$
\tilde{A}=\int_{-\infty}^{\infty} A e^{i \lambda s} d s
$$

satisfies

$$
\frac{d^{2} \tilde{A}}{d N^{2}}+2(\lambda+\gamma N) \tilde{A}=0
$$

with

$$
\tilde{A}=\int_{-\infty}^{\infty} e^{i S \lambda-\gamma^{2} S^{3} / 3} d S
$$

on $N=0$.

In order to write $\tilde{A}$ as an Airy function whose inverse transform can be taken along the real $\lambda$-axis, we need to define the cube root of $\left(-\gamma^{2}\right)$ in $(24)$ such that $A \rightarrow 0$ as $N \rightarrow+\infty$; for the case of the caustic we needed $A \rightarrow 0$ as $|N| \rightarrow \infty$ which constrained $A$ to be given by (12), but now the continuation 
of $A$ into $N<0$ will grow at infinity. The upshot is that $\tilde{A}$ is proportional to $A i\left(-2^{1 / 3} e^{\frac{2 i \pi}{3}(\lambda+\gamma N)}\right.$, the constant of proportionality being

$$
-2 \pi\left(\frac{2}{\gamma^{2}}\right)^{\frac{1}{3}} \frac{\operatorname{Ai}\left(-\left(\frac{2}{\gamma^{2}}\right)^{\frac{1}{3}} \lambda\right)}{\operatorname{Ai}\left(-2^{\frac{1}{3}} e^{\frac{2 i \pi}{3}} \lambda\right)}
$$

This leads to the famous Fock-Leontovich solution [8].

From our point of view it is especially interesting that $A$ can be written in Cartesian coordinates as a plane wave superposition

$$
A=\int_{\Gamma} \hat{p}(\lambda) e^{-i\left(y \lambda+\frac{x \lambda^{2}}{2}-\frac{\lambda^{3}}{3}\right)} d \lambda,
$$

where $\gamma=1$ for simplicity. This has been described most recently in [9]; here $\Gamma$ is no longer the real axis but is a suitable contour in the complex $\lambda$-plane and $\hat{p}$ is the Pekeris caret function [9], which is known as a "Fock-type integral" in the Russian literature, and can be most simply written as

$$
-\frac{e^{-\frac{2 i \pi}{3}}}{2 \pi} \int_{-\infty}^{\infty} e^{i \lambda t} \frac{\mathrm{Ai}(t)}{\operatorname{Ai}\left(t e^{\frac{2 i \pi}{3}}\right)} d t
$$

where $\lambda$ has negative imaginary part.

The existence of the explicit inversion of $\tilde{A}$ allows us to obtain a comprehensive description of the penumbral region that emerges as $x \rightarrow+\infty$. As described in [11] and, in terms of matched asymptotic expansions in [9], [10], this is achieved by taking suitable stationary-phase limits, and results in the birth of three thin-layer solutions of Helmholtz equation. These layers separate the illuminated region $y>0$ from the deep shadow region described above by a creeping wave field to lowest order. The upper and lower of the three layers are not, however, modelled by (2) but rather as the result of a Friedlander-Keller expansion. As shown in [10], this results in transition layers described by Pekeris functions of $(y / x)$, with an amplitude that decays as $x^{-\frac{1}{2}}$, while $y$ is of $O\left(k^{-\frac{1}{3}}\right)$ rather than the $k^{-\frac{2}{3}}$ scaling in the Fock-Leontovich region. Between these transition layers there is a classical shadow boundary in which $y$ is of $O\left(k^{-\frac{1}{2}}\right)$, as in Section 2.

With the next Section in mind, we conclude by considering how much of the penumbral structure and deep shadow could have been predicted from (16) without knowing the explicit transform solution. Two observations can be made:

a) As shown in [10], equation (2.46), a Friedlander-Keller expansion for the solution of (1) in $x>0, y<0$ is

$$
\phi \sim \frac{k^{-\frac{1}{6}} F(\rho)}{\tau^{\frac{1}{2}}} e^{i\left(k(\rho+\tau)+k^{\frac{1}{3}} v(\rho)\right)},
$$

where $F, v$ are unknown functions, $\rho$ is arclength along the scatterer to the point at which the creeping ray is shed and $\tau$ is the distance along that creeping ray. However, $F$ and $v$ can only be found by matching with the transform solution. 
b) While we can easily anticipate that there will be a shadow boundary as in Section 2.2 near the $x$-axis, we could also rescale $x$ and $y$ with $k^{\alpha}$ and $k^{2 \alpha}$ in (2) to give

$$
\frac{\partial^{2} A}{\partial y^{2}}+2 i k^{3 \alpha} \frac{\partial A}{\partial x}=0
$$

where $\frac{1}{3}>\alpha>\frac{1}{6}$ so as to be outside the deep shadow and the shadow boundary regions. Then a WKB expansion in which

$$
A \sim \hat{A} e^{i k^{3 \alpha} u}
$$

gives that $u=\frac{y^{2}}{2 x}$ and $\hat{A}=x^{-\frac{1}{2}} g\left(\frac{y}{x}\right)$ in conformity with the prediction at the end of the previous paragraph. Of course we cannot guess that $g$ is a Pekeris function, but we can see that $g(z) \sim O\left(\frac{1}{z}\right)$ as $z \rightarrow 0$ in order to match with a Fresnel integral.

We will now consider the problems of the diffraction of a caustic and a whispering gallery wave at an inflection point in the light of the above discussion.

\section{Thin Layers with Inflection Points}

The preceding Sections have shown how much easier it is to analyse thin layers in the absence of boundaries, so we will first consider a caustic whose curvature changes sign.

\subsection{Caustics}

Equation (12) shows how caustics with non-zero curvature can be simply modelled starting from (11). When we generalise the derivation to a caustic near $y+\frac{1}{3} \gamma x^{3}=0$, for which $\kappa \sim-2 \gamma x$ as $x \rightarrow 0$ we can, when $s$ is not too small, scale $n$ with $k^{-\frac{2}{3}}$ and retrieve (12). But as $s \rightarrow 0$, we must adopt the scalings that led to (16). We set $s=k^{-\frac{1}{5}} S, n=k^{-\frac{3}{5}} N$, where $S, N$ are new scaled variables distinct from those in Section 3; this gives, to lowest order,

$$
\frac{\partial^{2} A}{\partial N^{2}}+2 i \frac{\partial A}{\partial S}+4 \gamma N S A=0
$$

and we recall that $N$ is in the positive $y$-direction for all $S$. This equation has appeared many times in the literature, most recently in [20].

Since we are interested in incoming caustics in which, from Section 2.4 and in the light of the comments made at the end of Section 3.1,

$$
A \sim(-S)^{\frac{1}{3}} \operatorname{Ai}\left((-4 \gamma S)^{\frac{1}{3}} N\right)
$$


as $S \rightarrow-\infty$, the Fourier transform $\tilde{A}=\int_{-\infty}^{\infty} A e^{i \lambda N} d N$ satisfies

$$
-\lambda^{2} \tilde{A}+2 i \frac{\partial \tilde{A}}{\partial S}-4 i \gamma S \frac{\partial \tilde{A}}{\partial \lambda}=0
$$

with

$$
\tilde{A} \sim(4 \gamma)^{-\frac{1}{3}} \exp \left(\frac{i \lambda^{3}}{12 \gamma S}\right)
$$

as $S \rightarrow-\infty$; there is now no ambiguity over cube roots as there was after $(24)$. The characteristic equations for (31) are

$$
\frac{d S}{2 i}=\frac{d \lambda}{-4 i \gamma S}=\frac{d \tilde{A}}{\lambda^{2} \tilde{A}}
$$

so that the general solution is

$$
\tilde{A}=\exp \left(-\frac{i}{2}\left(\frac{\gamma^{2} S^{5}}{5}-\frac{2 \gamma\left(\lambda+\gamma S^{2}\right) S^{3}}{3}+\left(\lambda+\gamma S^{2}\right)^{2} S+F\left(\lambda+\gamma S^{2}\right)\right)\right)
$$

for some function $F$. However, (30), which holds for all sufficiently large negative values of $S$, implies that

$$
F(\zeta)=-\frac{8}{15 \gamma^{\frac{1}{2}}} \zeta^{\frac{5}{2}}-\frac{2 i}{3} \ln (4 \gamma)
$$

When we make the choice that $\left(\lambda+\gamma S^{2}\right)^{\frac{1}{2}}$ is positive when $\left(\lambda+\gamma S^{2}\right)>0$ and equal to $i\left|\lambda+\gamma S^{2}\right|^{\frac{1}{2}}$ when $\left(\lambda+\gamma S^{2}\right)<0$, the inversion integral for $\tilde{A}$ converges as long as we take the inversion contour $\Gamma$ along the positive real axis and just above the negative real axis in the $\lambda$-plane. Thus

$$
\begin{gathered}
A=\frac{1}{2 \pi i} e^{-i \gamma^{2} S^{5} / 5} \int_{\Gamma} \frac{1}{\left(\lambda+\gamma S^{2}\right)^{\frac{1}{2}}} \times \\
\exp \left(-i N \lambda-\frac{i}{2}\left(\frac{-8\left(\lambda+\gamma S^{2}\right)^{\frac{5}{2}}}{15 \gamma^{\frac{1}{2}}}+S\left(\lambda+\gamma S^{2}\right)^{2}-\frac{2 \gamma S^{3}}{3}\left(\lambda+\gamma S^{2}\right)\right)\right) d \lambda
\end{gathered}
$$

is the solution of (29) subject to (30), assuming uniqueness.

We note that when $S \rightarrow+\infty$ with $N=O(1)$, then, since $\left(\lambda+\gamma S^{2}\right)^{\frac{5}{2}} \sim$ $\gamma^{\frac{5}{2}} S^{5}, A$ will be proportional to $\operatorname{Ai}\left((4 \gamma S)^{\frac{1}{3}} e^{\frac{i \pi}{6}} N\right)$, and the amplitude decays exponentially in $S$. We will not analyse (4.1) further here apart from remarking that, as $|N| \rightarrow \infty, A$ decays to be of $O\left(|N|^{-\frac{5}{6}}\right)$ for $S \leq O\left(|N|^{-\frac{1}{2}}\right)$.

\subsection{Diffraction}

We now consider the open question that largely motivated the writing of this paper. This is the solution of (29) in $N>0$ and with $-\infty<s<\infty$, together with , for simplicity

$$
A=0 \text { on } N=0
$$


and,

$$
A \sim(-S)^{\frac{1}{3}} \exp \left(-\frac{3 i}{5 \gamma} 2^{\frac{1}{3}}(-\gamma S)^{\frac{5}{3}}\right) \operatorname{Ai}\left((-4 \gamma S)^{\frac{1}{3}} N+\sigma_{M}\right)
$$

as $S \rightarrow-\infty$, where $\sigma_{M}$ is the $M$ th zero of the Airy function.

This is the famous problem proposed by Popov [5] and it has subsequently been studied by many researchers from around the world (see, for example, some of the bibliography in [18]). Much research has been focused on the search for explicit solutions using weighted superpositions of elementary solutions such as (4),(5). In particular, Popov [15] has proposed a superposition of elementary solutions of the form

$$
\begin{gathered}
\frac{1}{\left(s-s_{0}\right)^{\frac{1}{2}}} \exp \left(\left(i \frac{\left(n-n_{0}\right)^{2}}{2\left(s-s_{0}\right)}\right) \times\right. \\
\exp \left(i n\left(\frac{1}{2} s_{0}\left(s-s_{0}\right)+\frac{1}{3}\left(s-s_{0}\right)^{2}\right)-i n_{0}\left(-\frac{s_{0}}{2}\left(s-s_{0}\right)-\frac{1}{6}\left(s-s_{0}\right)^{2}\right)\right) \times \\
\exp \left(i\left(-\frac{s^{2}}{24}\left(s-s_{0}\right)^{3}+\frac{s}{24}\left(s-s_{0}\right)^{4}-\frac{1}{90}\left(s-s_{0}\right)^{5}\right)\right)
\end{gathered}
$$

in unscaled coordinates $(s, n)$. Also the well-posedness of the problem has been proved in [14] and [13].

All this research has strongly suggested that the phase in any superposition of plane waves of the form (4) should include a fifth power of $\lambda$, as discussed in detail in [17], [18]. For the caustic described above, the phase contains a cubic power of $\lambda$ but only asymptotically as $S \rightarrow-\infty$, and it is possible that a fifth power would only emerge asymptotically in certain regions. ${ }^{2}$

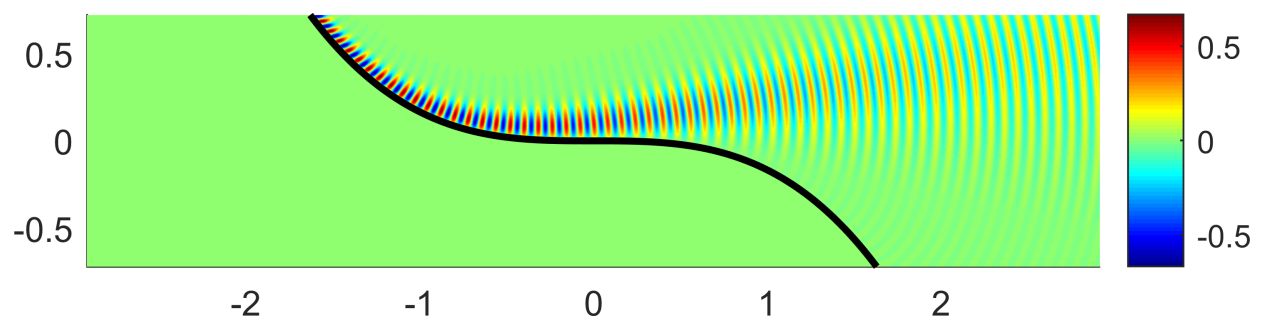

Figure 1: The $\mathrm{M}=1$ mode

The numerical investigations presented in [16] and [21] strongly suggest the generation of localised beams near the tangent at the inflection point, the number of beams being the number of zeros in the Airy function describing the incoming whispering gallery wave. Figures 1 and 2 are contour plots of the amplitude of the solution of (16), (36) and (37) with $m=3$ and $\gamma=\frac{1}{2}$ for the

\footnotetext{
${ }^{2}$ The birth of a caustic with an inflection point has been modelled in [1] using a phase involving fifth powers, but only at the expense of a singularity in the initial phase as a function of $y$.
} 


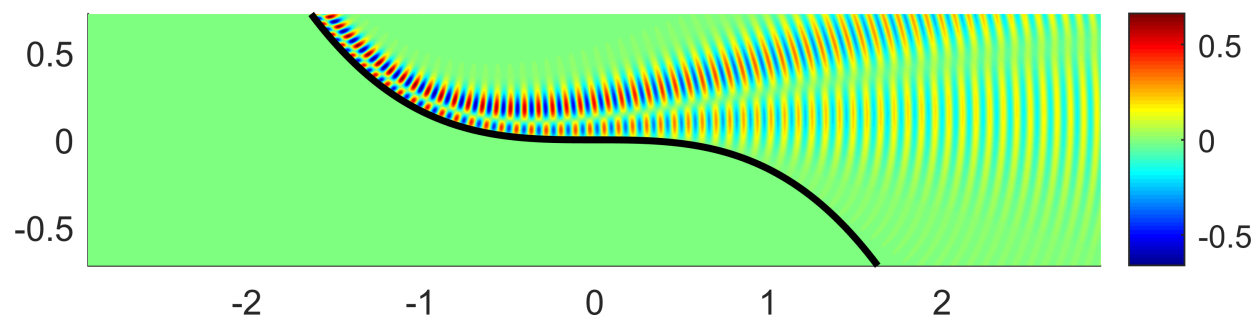

Figure 2: The $\mathrm{M}=2$ mode

parameter values $M=1,2$ in (37). These previously unpublished results were obtained by D.Hewett using a modern implementation of the finite-difference method used in [16] and [21]

As long as $s$ is not too small, the basic problem (16) with $m=3$ and $A=0$ on $n=0$ is, as in the case of the caustic above, amenable to a Friedlander-Keller ansatz in which $u(s)$ is proportional to $\sigma_{m} \int(-\kappa)^{\frac{2}{3}} d s$, as discussed in (2.29) of [10]. But when we write down the canonical inner problem in which $s=k^{-\frac{1}{5}} S$, $n=k^{-\frac{3}{5}} N$, we retrieve (29) with $N \geq 0$. Alas, no obvious transform in either $N$ or $S$ leads to a tractable problem in this case.

We conclude with two remarks and a conjecture.

(i) The fact that energy can be localised near the tangent at the inflection point has been demonstrated numerically in [16] and supported analyically in $[22]$.

(ii) If we anticipate some kind of beam formation near the $x$-axis then, motivated by $(28)$, we scale the problem in $(x, y)$ coordinates such that $x \sim O\left(k^{\alpha}\right), y \sim O\left(k^{3 \alpha}\right)$, the parabolic wave equation (2) becomes

$$
\frac{\partial^{2} A}{\partial y^{2}}+2 i k^{5 \alpha} \frac{\partial A}{\partial x}=0
$$

where, instead of the scaling after (28), $\frac{3}{5}>\alpha>\frac{1}{10}$. Now a WKB expansion $A \sim A^{*} e^{i k^{5 \alpha} u}$ gives, as at the end of Section 3.3, $u=\frac{y^{2}}{2 x}$, $A^{*}=x^{-\frac{1}{2}} G\left(\frac{y}{x}\right)$, where $G$ for the Popov problem is analogous to the Pekeris function for the Fock-Leontovich problem, and we expect $G(z)$ to be bounded as $z \rightarrow 0$. It has been conjectured that this function may be related to the function $G_{0}$ in equation (4.3) of [12] and to the function $A_{0}$ in equation (2.17) of [15], which was computed numerically in [16].

(iii) Finally, based on all that has been written above, we make the speculative conjecture that the solution of the Popov problem will involve a localisation near the $x$-axis, possibly of the form of a sum of "Gaussian 
beams"

$$
\phi \sim \sum_{j=1}^{M} c_{j} \frac{e^{-\frac{\left(y-y_{j}\right)^{2}}{2\left(i x+X_{0}\right)}}}{\sqrt{i x+X_{0}}}
$$

where $x<<X_{0},\left|y_{j}\right|<<k^{-\frac{1}{2}}$ and $M$ is the number of zeros in the amplitude of the incoming creeping field.

\section{Conclusion}

This brief review has emphasised the diversity of high-frequency wave propagation problems that can be modelled by the parabolic wave equation(2), many of which are catalogued in [19]. We have concentrated on the derivation of thin layer solutions of the Helmholtz equation using asymptotic analysis, but we have not discussed the many important implications it may have for numerical computations, and vice versa; a clear example of the latter is presented in [25], while Figures 1 and 2 were an invaluable stimulus and motivation for much of the current work.

The main implications that have been discussed are

(i) the use of superpositions of elementary solutions of (2) in Cartesian coordinates,

(ii) the catalogue of phenomena that can be described when (2) is written in curvilinear coordinates, for which pioneering work was done in [8] and [9] for regions with parabolic boundaries,

(iii) the need for further analytical and numerical investigation of the "Popov problem", concerning which the literature only allows us to make conjectures, albeit fairly confident ones. Of particular practical interest is the amplitude of the outgoing creeping field.

\section{Acknowledgements}

Our thanks go to Dr D. P. Hewett and especially to Professor V. P. Smyshlyaev for many perspicacious comments, to Dr Colin Sillence of BAe Systems who initiated UK research in the Popov problem 30 years ago, and to Dr Hilary Ockendon for help with preparing the manuscript.

\section{References}

[1] J. R. Ockendon, R. H. Tew, Thin-layer solutions of the Helmholtz and related equations, SIAM Rev. 54(1) (2012) 3-51.

[2] R. Buchal, J. B. Keller, Boundary Layer Problems in Diffraction Theory, Comm.Pure Appl. Math. 13 (1960) 85-144. 
[3] R. H. Tew, Friedlander-Keller ray expansions and scalar wave reflection at canonically perturbed boundaries, Euro. J. Appl. Math. 30 (2019) 1-22.

[4] P. J. Olver, Applications of Lie Groups to Partial Differential Equations, Springer (1991).

[5] M. M. Popov, The problem of whispering gallery waves in a neighbourhood of a simple zero of the effective curvature of the boundary. J.Sov.Math. 11 (1979) 791-797.

[6] H. M. Nussenszveig, High-frequency scattering by an impenetrable sphere. Annals of Physics 34 (1965) 23-95.

[7] D. S. Jones, Acoustic and electromagnetic waves, Oxford University Press (1986).

[8] M. S. Leontovich, V. A. Fock, Solution of the problem of propagation of electromagnetic waves along the earth's surface by the method of the parabolic equation, J.Phys.USSR 10 (1946) 13-24.

[9] D. P. Hewett, Tangent ray diffraction and the Pekeris caret function, Wave Motion 57 (2015) 257-267.

[10] R. H. Tew, S. J. Chapman, J. R. King, J. R. Ockendon, B. J. Smith, I. Zafarullah, Scalar wave diffraction by tangent rays, Wave Motion 32 (2000) 363-380.

[11] V. M. Babich, V. S. Buldyrev, Short Wavelength Diffraction Theory, Springer, Berlin ((1991).

[12] V. M. Babich, V. P. Smyshlyaev, Scattering Problems for the Schrödinger equation in the case of a potential linear in time and coordinate I: Asymptotics in the shadow zone, J.Sov.Math 32 (1986) 103-111.

[13] V. M. Babich, V. P. Smyshlyaev, The Scattering problem for the Schrödinger equation with a potential linear in time and space II: Correctness, smoothness, behaviour of the solution at infinity. J.Sov.Math. $\mathbf{3 8}$ (1987) 1562-1576.

[14] M. M. Popov, Correctness of the problem of Whispering Gallery Waves in a neighbourhood of points of zero curvature of the boundary, J.Sov.Math 19 (1982) 1487-1493.

[15] M. M. Popov, Whispering Gallery Waves in a neighbourhood of an Inflection Point at the Boundary. Asymptotics of the Wave Field as $t \rightarrow \infty$, J.Sov.Math 32(1986) 215-219.

[16] M. M. Popov, V. G. Krasavin, Direction Diagram of Radiation in the problem of an Inflection Point of the Boundary, J,Sov.Math 32 (1986) 215-219. 
[17] A. Y. Kazakov, Special Function related to the concave-convex boundary problems of the diffraction theory, J.Phys.A:Math.Gen 36 (2003) 41274141.

[18] D. P. Hewett, J. R. Ockendon, V. P. Smyshlyaev, Contour integral solution of the parabolic wave equation, Wave Motion 84(2019) 90-109.

[19] V. M. Babich, N. Y. Kirpichnikova, The boundary Layer Method in Diffraction Problems, Springer-Verlag, 1979.

[20] A. Ya. Kazakov, "Separation of variables" in the Model Problems of the Diffraction Theory. A Formal Scheme. J.Math.Sci 243 (2019) 715-725.

[21] M. M. Popov and I. Pshenchik, Numerical solution of the problem on whispering gallery waves in a neighborhood of a simple zero of the effective curvature of the boundary, J. Sov. Math. (now J. Math. Sci.), 11 (1979), 797-804.

[22] V. P. Smyshlyaev, Concentration of the solution near a limit ray in the neighbourhood of an Inflection point of the boundary, J. Sov. Math., 55 (1991), 1757-1760.

[23] D. Ludwig, Wave Propagation near a Smooth Caustic, Bull.Am.Math.Soc. 73 (1965), 776-779.

[24] Yu. A. Kravtsov, A modification of the geometrical optics method, Radiofizik 7 (1964), 1049-1056 (in Russian).

[25] D. P. Hewett, Shadow boundary effects in hybrid numerical-asymptotic methods for high-frequency scattering, Euro. J. Appl. Math. 26 (2015), 773-793. 